\title{
The box and Hausdorff dimension of self-affine sets
}

\author{
TIM BEDFORD ${ }^{1}$ AND MARIUSZ URBAŃSKI ${ }^{2}$ \\ ${ }^{1}$ Department of Mathematics and Informattcs, Delft University of Technology, \\ PO Box 356, 2600 AJ Delft, The Netherlands \\ ${ }^{2}$ Intytut Matematykı, Uniwersytet M Kopernika, ul Chopına 12/18, 87-100 Toruń, \\ Poland
}

(Recelved 28 November 1988 and revised 22 June 1989)

\begin{abstract}
Under a natural assumption the Hausdorff dimension of a measure $\mu$ canonically associated with a given self-affine set is computed A simplified proof of Bowen's formula for the box dimension of self-affine sets proved earlier is given A condition for the box dimension and Hausdorff dimension to be equal is proven, and a collection of examples in which this condition can be checked is discussed
\end{abstract}

\section{Introduction}

In this paper we consider the box dimension (or 'capacity') and Hausdorff dimension of certain self-affine sets which include those studied in [Be2] and in $\S 6$ of [PU] Bedford [Be2] calculated the box dimension of some self-affine connected curves and obtained a formula involving the topological pressure of a certain function This formula is analogous to that of Bowen [Bo2] for the Hausdorff dimension of self-sımılar sets (see also Manning and McCluskey's formula [MM] in a slightly different context, and also [Be1] for the connection with box dimension) Here we give a sımplified proof of the formula for the box dimension of self-affine sets Our approach is based only on the theory of Gibbs states presented in [Bol] and does not involve the more advanced thermodynamic formalism of [R] and the 'singularity spectrum' results of [BR]

The main part of this paper is stimulated by a conjecture made in the preprint version of [Be2] The box dimension formula obtained in [Be2] states that the box dimension of a self-affine curve $E \subset \mathbb{R}^{2}$ is given by the unique real $t+1$ such that the topological pressure $P\left(t f_{W}+f_{H}\right)=0$ where $f_{W}$ and $f_{H}$ are functions measuring the scaling structure of $E$ If $\mu$ is the equilibrium state for $t f_{W}+f_{H}$ and $\pi_{*} \mu$ an associated measure on $E$ then we can state the conjecture from [Be2] as follows the Hausdorff dimension of $E$ is equal to the box dimension of $E$ if and only if $H D\left(p_{H} \pi_{*} \mu\right)=1$, where $p_{H} \mathbb{R}^{2} \rightarrow \mathbb{R}$ is orthogonal projection onto the $y$-axis For certain self-affine sets which generalize the so-called limit Radamacher functions considered in [PU] we calculate the Hausdorff dimension of the measure $\pi_{*} \mu$ The formula obtained permits (for this class of sets) to give a positive answer for one 
direction of this conjecture and some positive partial contributions to the other direction In the last section of this paper we describe two classes of self-affine sets for which we are able to verify the assumptions we make to obtain our results

We now recall some of the general notions and results used in this paper

If $A$ is a subset of a metric space $(X, \rho)$ then the box dimension of $A$ is defined as follows Let $N(A, \varepsilon)$ denote the minimum number of balls of radius $\varepsilon>0$ needed to cover $A$ We set

$$
\begin{aligned}
& \underline{D}_{B}(A)=\liminf _{\varepsilon \rightarrow 0} \frac{\log N(A, \varepsilon)}{-\log \varepsilon}, \\
& \bar{D}_{B}(A)=\operatorname{limsuf}_{\varepsilon \rightarrow 0} \frac{\log N(A, \varepsilon)}{-\log \varepsilon}
\end{aligned}
$$

If $\underline{D}_{B}(A)=\bar{D}_{B}(A)$ then the common value is called the box dimension of $A$ and is denoted by $D_{B}(A)$ For the definition and basic properties of Hausdorff dimension, which we denote by $H D$, we refer the reader to the book by Falconer [Fa] The Hausdorff dimension and box dimension are related by

$$
H D(A) \leq \underline{D}_{B}(A) \leq \bar{D}_{B}(A)
$$

If $\mu$ is a Borel probability measure on $X$ then the Hausdorff dimension of $\mu$ is defined as

$$
H D(\mu)=\operatorname{lnf}\{H D(Y) \quad Y \subset X \text { and } \mu(Y)=1\}
$$

In order to estımate the Hausdorff dimension of a Borel probability measure on a Euclidean space we shall rely on the following well known result (see [PU])

Frostman's LemMA If for $\mu$-a e $x$ we have

$$
\delta_{1} \leq \lim _{\varepsilon \rightarrow 0} \frac{\log \mu(B(x, \varepsilon))}{\log \varepsilon} \leq \delta_{2},
$$

where $B(x, \varepsilon)$ is the ball of radius $\varepsilon$ around $x$, then $\delta_{1} \leq H D(\mu) \leq \delta_{2}$

If $f, g \quad X \rightarrow \mathbb{R}$ are two real-valued functions then we shall say that $f$ and $g$ are boundedly equivalent and write $f \simeq g$ if there is a constant $C \geq 1$ such that

$$
C^{-1} g(x) \leqslant f(x) \leqslant C g(x) \text { for every } x \in X
$$

Note that $\simeq 1$ an equivalence relation and that $a f \simeq f$ for every $a>0$

\section{The construction of self-affine sets}

In this section we recall some of the facts and definitions from [Be2] that we need here They are formulated in a slightly more general settıng which enables us to deal with disconnected self-affine sets as well At the end of this section we present another proof of the Bowen dimension formula for self-affine sets first proved in [Be2] Note that our notation does not coincide everywhere with that of [Be2]

We put $I=[0,1]$ and consider orientation preserving, contractive $C^{1+\varepsilon}$ diffeomorphisms $\varphi, I \times \mathbb{R} \rightarrow I \times \mathbb{R}$ (for $0 \leq \imath<k$ ) satısfying the following properties $\varphi_{1}(x, y)=\left(\psi_{1}(x), \tau_{1}(x, y)\right), 0 \leq t<k$ for some differentiable functions $\psi_{1} I \rightarrow I$ 
and $\tau_{1} I \times \mathbb{R} \rightarrow \mathbb{R}$ such that

$$
A=\sup \left\{\psi_{\prime}^{\prime}(x) \quad x \in I, 0 \leq \imath<k\right\}<1
$$

and

$$
B=\sup \left\{\frac{\partial}{\partial y} \tau_{l}(x, y)(x, y) \in I \times \mathbb{R}, 0 \leq \imath<k\right\}<1,
$$

the fixed points of $\varphi_{0}$ and $\varphi_{k-1}$ are $\left(0, y_{0}\right)$ and $\left(1, y_{k-1}\right)$ respectively for some $y_{0}, y_{k-1} \in \mathbb{R}$,

and

$$
\psi_{i}(1)=\psi_{\imath+1}(0) \text { for every } 0 \leq \imath<k-1
$$

We also define $a$ and $b$ by

$$
a=\inf \left\{\psi_{1}^{\prime}(x) \quad x \in I, 0 \leq \imath<k\right\}>0
$$

and

$$
b=\inf \left\{\frac{\partial}{\partial y} \tau_{1}(x, y)(x, y) \in I \times \mathbb{R}, 0 \leq 1<k\right\}>0
$$

The constants $a, b, A$ and $B$ will be used as defined here throughout the paper

By a result of Hutchinson [H] there is a unique compact non-empty set $E$ such that $E=\bigcup_{i=0}^{k-1} \varphi_{1}(E)$ Following [Be2] we shall call such sets self-affine Let $\Sigma=$ $\prod_{1}^{\infty}\{0, \quad, k-1\}$ For $\underline{x}=\left(x_{1}, x_{2}, \quad\right) \in \Sigma$ we set $\underline{x}(n)=\left(x_{1}, \quad, x_{n}\right),(n \geq 1)$, and write

$$
\varphi_{\underline{x}(n)}=\varphi_{x_{1}} \circ \quad \circ \varphi_{x_{n}}, \quad \psi_{\underline{x}(n)}=\psi_{z_{1}} \circ \quad \circ \psi_{x_{n}}
$$

and

$$
\pi(\underline{x})=\bigcap_{n=1}^{\infty} \varphi_{\underline{x}(n)}(E) \quad \tilde{\pi}(\underline{x})=\bigcap_{n=1}^{\infty} \psi_{\underline{x}(n)}(E)
$$

We shall often identify the finite sequence $\underline{x}(n)$ with the subset $\{\underline{y} \in \Sigma \quad \underline{y}(n)=\underline{x}(n)\} \subset$ $\Sigma$

Since $\varphi$ and $\psi$ are strict contractions and the families

$$
\left\{\varphi_{\underline{x}(n)}(E)\right\}_{n=1}^{\infty}, \quad\left\{\psi_{\underline{x}(n)}(I)\right\}_{n=1}^{\infty}
$$

are decreasing in $n$, the sets $\pi(\underline{x})$ and $\tilde{\pi}(\underline{x})$ are singletons Furthermore $\pi(\underline{x}) \in E$ since $\varphi_{\underline{x}(n)}(E) \subset E$ for all $n \geq 1$ We have thus constructed maps

$$
\pi \Sigma \rightarrow E \text { and } \tilde{\pi} \Sigma \rightarrow I \text {, }
$$

which are contınuous since $\operatorname{diam}\left(\varphi_{\underline{x}(n)}(E)\right)$ and $\operatorname{diam}\left(\psi_{\underline{x}(n)}(I)\right)$ converge to 0 uniformly (in fact exponentially) fast The two maps are surjective because $E=$ $\bigcup_{i=0}^{k-1} \varphi_{i}(E)$ and $I=\bigcup_{t=0}^{k-1} \psi_{t}(I)$ The following properties are also easy to see $\pi\left(l, x_{1}, x_{2}, \quad\right)=\varphi_{1}\left(x_{1}, x_{2}, \quad\right)$ and $\tilde{\pi}\left(l, x_{1}, x_{2}, \quad\right)=\psi_{1}\left(x_{1}, x_{2}, \quad\right)$ 
$\pi$ and $\tilde{\pi}$ are injective except that given $x_{1}, \quad, x_{n}$ with $x_{n} \neq k-1$ the two sequences $\left(x_{1}, \quad, x_{n}, k-1, k-1, \quad\right)$ and $\left(x_{1}, \quad, x_{n}, 0,0, \quad\right)$ have the same image under $\tilde{\pi}$ and may have the same image under $\pi$

An easy consequence of $(24)$ is

$$
\varphi_{\underline{x}(n)}(E)=\pi(\underline{x}(n)) \quad \text { and } \quad \psi_{\underline{x}(n)}(I)=\tilde{\pi}(\underline{x}(n))
$$

Finally, we define $p_{W} I \times \mathbb{R} \rightarrow I$ and $p_{H} I \times \mathbb{R} \rightarrow \mathbb{R}$ to be the standard projection maps $(x, y) \mapsto x$ and $(x, y) \mapsto y$ respectively For a subset $D$ of $\mathbb{R}$ we denote the diameter by $|D|$ and the Lebesgue measure by $l(D)$ For $K \subset \mathbb{R}^{2}$ we put $|K|_{W}=$ $\left|p_{W}(K)\right|,|K|_{H}=\left|p_{H}(K)\right|, l_{W}(K)=l\left(p_{W}(K)\right)$ and $l_{H}(K)=l\left(p_{H}(K)\right)(W$ and $H$ stand for 'width' and 'height' respectively)

As $p_{W} \varphi_{t}=\psi_{i} p_{W}$, for $0 \leq l<k$, we obtain

$$
p_{W} \circ \pi=\tilde{\pi}
$$

The following lemma says that $E$ is almost the graph of a function

LEMMA 1 For all $x \in I$ outside a countable set $Z \subset I$, each line $\{x\} \times \mathbb{R}$ contains exactly one point of $E$

Proof Let $X_{0}=\left\{\underline{x} \in \Sigma x_{n}=0\right.$ for all large $\left.n\right\}, X_{k-1}=\left\{\underline{x} \in \Sigma x_{n}=k-1\right.$ for all large $n$, and let $Z=\tilde{\pi}\left(X_{0} \cup X_{k-1}\right)$ Now $Z$ is countable since $X_{0}$ and $X_{k-1}$ are countable By (25), card $\tilde{\pi}^{-1}(x)=1$ for every $x \in I \backslash Z$, and so (27) implies that card $(E \cap\{x\} \times$ $\mathbb{B})=1$ This proves the lemma

Condition (2 1) implies that

$$
D \varphi_{l}(x, y)=\left(\begin{array}{cc}
\psi_{1}^{\prime}(x) & 0 \\
\frac{\partial}{\partial x} \tau_{l}(x, y) & \frac{\partial}{\partial y} \tau_{\imath}(x, y)
\end{array}\right) \quad 0 \leq l<k
$$

From now on we shall assume that

$$
\lambda=\sup \left\{\frac{\psi_{\imath}^{\prime}(x)}{\frac{\partial}{\partial y} \tau_{l}(x, y)}(x, y) \in I \times \mathbb{R}, 0 \leq l<k<1,\right\}
$$

which means that the maps $\varphi$, have a sharper contraction horizontally than vertically We define two functions which together measure the contraction rates of the $\varphi_{1}$ Let $f_{W}, f_{H} \Sigma \rightarrow \mathbb{R}$ be given by

$$
f_{W}(\underline{x})=\log \psi_{x_{t}}^{\prime}(\tilde{\pi}(\sigma x)) \quad f_{H}(\underline{x})=\log \frac{\partial}{\partial y} \tau_{x_{1}}(\pi(\sigma x))
$$

(note that these functions are minus the corresponding functions in [Be2], but that they have the same sign as the corresponding function in [Bo2] and [MM]) As the functions $\sigma, \pi, \tilde{\pi},(\partial / \partial y) \tau_{x_{1}}$ and $\psi_{x_{1}}^{\prime}$ are contınuous, both $f_{W}$ and $f_{H}$ are contınuous It is not difficult to check (see [Be2]) that they are actually Holder continuous For $f=f_{W}$ or $f_{H}$ and $n \geq 1$ we denote the sum $\sum_{i=0}^{n-1} f\left(\sigma^{\prime} \underline{x}\right)$ by $S_{n} f(\underline{x})$ 
We can reformulate Lemma 3, Lemma 5 and Proposition 8 of [Be2] as follows

LEMMA 2 For every $n \geq 1$ and $\underline{x}, \underline{y} \in \Sigma$ with $\underline{x}(n)=\underline{y}(n)$ we have

$$
\exp S_{n} f_{W}(\underline{x}) \simeq \exp S_{n} f_{W}(\underline{y}) \text { and } \exp S_{n} f_{H}(\underline{x}) \simeq \exp S_{n} f_{H}(\underline{y})
$$

LEMMA 3 For every $n \geq 1$ and $\underline{x} \in \Sigma$,

$$
|\tilde{\pi}(\underline{x}(n))|=\left|\psi_{\underline{x}(n)}(I)\right|=\left|\varphi_{\underline{x}(n)}(E)\right|_{W} \approx \exp S_{n} f_{W}(\underline{x})
$$

LEMMA 4 For every $n \geq 1$ and $\underline{x} \in \Sigma$,

$$
|\pi(\underline{x}(n))|_{H}=\left|\varphi_{\underline{x}(n)}(E)\right|_{H} \simeq \exp S_{n} f_{H}(\underline{x})
$$

An extra condition is needed for Lemma 4 to hold as stated This is because there is a degenerate case in which $E$ is a differentiable manifold However, this happens If and only if the strong stable manifolds of the maps $\varphi_{1}$ all coincide (see [Be $\geqslant 2$ ] for more details) and we shall assume that this highly non-generic possibility does not occur We remark also that the proof of (our) Lemma 4 given in [Be2] required the existence of sets $C \subset E$ with $|C|_{H} /|C|_{W}$ arbitrarly large This is true in the disconnected case since for some $i$ we have $\varphi_{I+1}\left(\left(0, y_{0}\right)\right) \neq \varphi_{i}\left(\left(0, y_{k-1}\right)\right)$ (using the notation of (2 2)) -for otherwise [Be2] shows that $E$ is connected Hence the set $C=\left\{\varphi_{i+1}\left(\left(0, y_{0}\right)\right), \varphi_{i}\left(\left(0, y_{k-1}\right)\right)\right\}$ has $|C|_{H}>0$, but $|C|_{W}=0$ by (2 3) With this remark, the proofs of the above lemmas work in exactly the same way as those in [Be2]

The main technical tools we use in this paper are the notions of topological pressure and Gibbs states Topological pressure with respect to $\sigma \Sigma \rightarrow \Sigma$ is an operator on the space of real-valued continuous function on $\Sigma$ It satisfies a var1atıonal principle relatıng it to measure theoretıc entropy,

$$
P(g)=\sup \left\{h_{\mu}(\sigma)+\int g d \mu\right\} \quad g \in C(\Sigma),
$$

where the supremum is taken over $\sigma$-invariant Borel probability measures A measure taking the supremum is called an equilibrium state for $g$ When $g$ is Holder contınuous there is a unique equilibrium state $\mu$ for $g$, which is a Gibbs measure This means that for all $\underline{x} \in \Sigma$ and $n \geq 0$ we have

$$
\mu(\underline{x}(n)) \simeq \exp \left\{S_{n} g(\underline{x})-n P(g)\right\}
$$

(see [Bo1], $14 \mathrm{pp}$ 9-10) More information about pressure and Gibbs states can be found in [Bo1] The formula for box dimension of $E$ established in [Be2] involves the zero of the function $s \mapsto P\left(s f_{W}+f_{H}\right)$ Our assumptions that $0<a, b, A, B<1$ imply that there is a unique $t \in \mathbb{R}$ with $P\left(t f_{W}+f_{H}\right)=0$ Furthermore we have the following bound on the value of $t$

LEMMA 5 The unique real number $t$ defined by $P\left(t f_{W}+f_{H}\right)=0$ satisfies $0<t<1$

Proof We first show that $t>0$ Let $\lambda$ be the equilibrium state for the function $f_{W}$ By (29) and Lemma 3 we have

$$
\lambda(\underline{x}(n)) \sim \exp \left(S_{n} f_{W}(\underline{x})-n P\left(f_{W}\right)\right) \sim|\tilde{\pi}(\underline{x}(n))| \exp \left(-n P\left(f_{W}\right)\right)
$$


for every $n \geq 1$ and $x \in \Sigma$ Therefore

$$
1=\sum_{\underline{x}(n)} \lambda(\underline{x}(n)) \sim \sum_{x(n)}|\tilde{\pi}(\underline{x}(n))| \exp \left(-n P\left(f_{W}\right)\right)=\exp \left(-n P\left(f_{W}\right)\right)
$$

for every $n \geq 1$, which implies that $P\left(f_{W}\right)=0$ By (28) and (21) we have $f_{W}<f_{H}<0$, which gives $0=P\left(f_{W}\right)<P\left(f_{H}\right)$ As the function $s \mapsto P\left(s f_{W}+f_{H}\right)$ is strictly decreasing we obtain $t>0$

To see that $t<1$, let $\nu$ be the equilibrium state for the function $f_{W}+f_{H}$ As $P\left(f_{W}\right)=0$, the variational principle for pressure implies that

$$
\begin{aligned}
P\left(f_{W}+f_{H}\right) & =h_{\nu} \sigma+\int f_{W} d \nu+\int f_{H} d \nu \\
& \leq P\left(f_{W}\right)+\int f_{H} d \nu \\
& =\int f_{H} d \nu<0
\end{aligned}
$$

Th1s together with strict monotonicity of $s \mapsto P\left(s f_{W}+f_{H}\right)$ shows that $t<1$

DEFINITION We say that $E$ sattsfies the Darboux property if the set $p_{H}\left(\varphi_{x(n)}(E)\right)$ is connected for every $n \geq 1$ and $\underline{x} \in \mathbf{\Sigma}$

We end this section with another proof of the Bowen formula established in [Be2]

TheOREM 6 If E satisfies the Darboux property then $D_{B}(E)=t+1$

Proof Let $\mu$ be the equilibrium state for the function $t f_{W}+f_{H}$, and fix $\varepsilon>0$ Since $0<a \leq A<1$ we can find a finite number of points $\underline{x}^{1}, \quad \underline{x}^{p} \in \Sigma$ and integers $n_{1}, \quad, n_{p} \geq 1$ such that

$$
\begin{gathered}
\bigcup_{j=1}^{p} \tilde{\pi}\left(\underline{x}^{j}\left(n_{j}\right)\right)=I, \\
\operatorname{lnt}\left(\tilde{\pi}\left(\underline{x}^{j}\left(n_{j}\right)\right)\right) \cap \operatorname{lnt}\left(\tilde{\pi}\left(\underline{x}^{\prime}\left(n_{\imath}\right)\right)\right)=\varnothing \quad \text { for } 1 \leq \imath \neq j \leq p,
\end{gathered}
$$

and

$$
\varepsilon a \leq\left|\tilde{\pi}\left(\underline{x}^{J}\left(n_{J}\right)\right)\right| \leq \varepsilon \text { for } 1 \leq J \leq p
$$

For each $J$ define $q=\left[\left|\pi\left(\underline{x}^{j}\left(n_{j}\right)\right)\right|_{H} \cdot\left|\tilde{\pi}\left(\underline{x}^{j}\left(n_{j}\right)\right)\right|^{-1}\right]+1$ Clearly we can find $u_{1}, \quad, u_{q_{j}} \in \mathbb{R}$ (depending on $j$ ) such that $\tilde{\pi}\left(\underline{x}^{j}\left(n_{j}\right)\right) \times\left[u_{1}, u_{q_{j}}\right] \supset \pi\left(\underline{x}^{j}\left(n_{j}\right)\right)$ and $u_{t+1}-u_{\imath}=\left|\tilde{\pi}\left(\underline{x}^{j}\left(n_{j}\right)\right)\right|$ for $1 \leq \imath<q$, By Lemmas 3 and 4 we have

$$
\begin{aligned}
q_{j} & \simeq\left|\pi\left(\underline{x}^{j}\left(n_{j}\right)\right)\right|_{H}\left|\tilde{\pi}\left(\underline{x}^{j}\left(n_{j}\right)\right)\right|^{-1} \\
& \simeq \exp \left(S_{n_{1}} f_{H}\left(\underline{x}^{j}\right)\right) \quad \exp \left(-S_{n_{t}} f_{W}\left(\underline{x}^{j}\right)\right) \\
& =\exp \left(S_{n_{j}}\left(f_{H}-f_{W}\right)\left(\underline{x}^{j}\right)\right)
\end{aligned}
$$

Clearly $N(E, \varepsilon) \leq \sum_{j=1}^{p} q_{j}$ Furthermore as $E$ satisfies the Darboux property and any ball of radius $\frac{1}{2} \alpha \varepsilon$ can intersect at most 4 rectangles of the form $\tilde{\pi}\left(\underline{x}^{j}\left(n_{j}\right)\right) \times$ $\left[u_{i}, u_{i+1}\right]$, we must have $N\left(E, \frac{1}{2} a \varepsilon\right) \geq \frac{1}{4} \sum_{j=1}^{p} q$, 
Since $\mu$ is the equilibrium state for $\mathrm{tf}_{\mathrm{W}}+\mathrm{f}_{\mathrm{H}}$ where $P\left(t f_{W}+f_{H}\right)=0$, 1t follows from (29) that for any $\varepsilon>0$

$$
\begin{aligned}
N(E, \varepsilon) & \simeq \sum_{j=1}^{p} q_{j} \simeq \sum_{j=1}^{p} \exp \left(S_{n_{j}}\left(f_{H}-f_{W}\right)\left(\underline{x}^{\jmath}\right)\right) \\
& =\sum_{j=1}^{p} \exp \left(S_{n_{j}}\left(t f_{W}+f_{H}\right)\left(\underline{x}^{J}\right)\right) \exp \left(-S_{n_{j}}(1+t) f_{W}\left(\underline{x}^{j}\right)\right) \\
& \simeq \sum_{j=1}^{p} \mu\left(\underline{x}^{J}\left(n_{j}\right)\right)\left|\tilde{\pi}\left(\underline{x}^{J}\left(n_{j}\right)\right)\right|^{-(1+t)} \\
& \simeq \sum_{j=1}^{p} \tilde{\pi}_{*} \mu\left(\tilde{\pi}\left(\underline{x}^{J}\left(n_{\jmath}\right)\right)\right) \varepsilon^{-(1+t)}=\varepsilon^{-(1+t)}
\end{aligned}
$$

Therefore $D_{B}(E)=t+1$ and the theorem is proved

REMARK (1) The Darboux property was not used to obtain the estimate

$$
N(E, \varepsilon) \leq \sum_{j=1}^{p} q_{j}
$$

and so the inequality $D_{\mathrm{B}}(E) \leq t+1$ is true for any self-affine set $E$

(11) The proof of $D_{B}(E)=t+1$ given in [Be2] establishes the result via a variational principle This principle also contains information about the number of boxes required to cover certain subsets of $E$

\section{The dimension of $E$ and $\boldsymbol{\pi}_{*} \boldsymbol{\mu}$}

From now on we make the additional assumption that

$$
\frac{\partial}{\partial x} \tau_{\imath}(x, y) \equiv 0 \quad \text { for } 0 \leq \imath<k
$$

and will say that $E$ has an invariant weak foliation We are aiming towards conditions under which the Hausdorff dimension of $E$ is equal to $t+1$, and shall do this via a study of the Hausdorff dimension of $\pi_{*} \mu$ If $E$ has an invariant weak foliation then each $\varphi_{1}, I \times \mathbb{R} \rightarrow I \times \mathbb{R}$ can be expressed in the form

$$
\varphi_{1}(x, y)=\left(\psi_{1}(x), \tau_{1}(y)\right) \text { where } \tau_{1} \mathbb{R} \rightarrow \mathbb{R}
$$

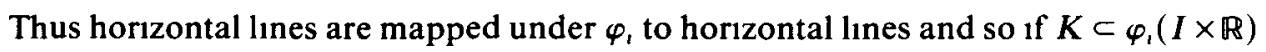
is a rectangle with sides parallel to the $x$ and $y$-axes then $\varphi_{1}^{-1}(K)$ is also such a rectangle - in future all rectangles used in the proofs will be of this form It follows from Lemma 4 that if $\underline{x} \in \Sigma, n \geq 1$ and $K \subset I \times \mathbb{R}$ has the property that $p_{H}(K) \subset$ $p_{H} \varphi_{\underline{x}(n)}(E)$ then

$$
\left|\varphi_{\underline{x}(n)}^{-1}(K)\right|_{H} \simeq|K|_{H} \exp \left(-S_{n} f_{H}(\underline{x})\right)
$$

In particular we obtain the following

LEMMA 7 Let

$$
\begin{aligned}
& K_{+}(\underline{x}, n)=\tilde{\pi}(\underline{x}(n)) \times\left[p_{H} \pi(\underline{x}), p_{H} \pi(\underline{x})+\frac{1}{2}|\tilde{\pi}(\underline{x}(n))|\right], \\
& K_{-}(\underline{x}, n)=\tilde{\pi}(\underline{x}(n)) \times\left[p_{H} \pi(\underline{x})-\frac{1}{2}|\tilde{\pi}(\underline{x}(n))|, p_{H} \pi(\underline{x})\right],
\end{aligned}
$$


and $K(\underline{x}, n)=K_{+}(\underline{x}, n) \cup K_{-}(\underline{x}, n)$ Then for any $\underline{x} \in \Sigma$ and $n \geq 1$ we have

$$
\begin{aligned}
\left|\varphi_{\underline{x}(n)}^{-1}\left(K_{+}(\underline{x}, n)\right)\right|_{H} & \simeq\left|\varphi_{\underline{x}(n)}^{-1}\left(K_{-}(\underline{x}, n)\right)\right|_{H} \simeq\left|\varphi_{\underline{x}(n)}^{-1}(K(\underline{x}, n))\right|_{H} \\
& \simeq|\tilde{\pi}(\underline{x}(n))| \exp \left(-S_{n} f_{H}(\underline{x})\right)
\end{aligned}
$$

As before we let $\mu$ be the equilibrium state for the function $t f_{W}+f_{H}$, and we now take $\nu$ to be the Borel probability measure on $\Sigma$ given by Ruelle's Perron-Frobenius theorem (see [Bol] $17 \mathrm{p}$ 14) for $t f_{W}+f_{H}$ The two measures $\mu$ and $\nu$ are equivalent with continuous never vanishing densities It follows from this theorem that for $K \subset \varphi_{\imath}(I \times \mathbb{R})(0 \leq \imath<k)$ we have

$$
\pi_{*} \nu\left(\varphi_{i}^{-1}(K)\right)=\int_{K} \exp \left(-t f_{W}-f_{H}\right) d\left(\pi_{*} \nu\right) \geq \pi_{*} \nu(K)
$$

We consider $\pi_{*}, \nu$ here as a measure defined on $I \times \mathbb{B}$ with the property that $\left.\pi_{*} \nu(I \times \mathbb{R}) \backslash E\right)=0$ Therefore, using Lemma 2, we get the following,

LEMMA 8 For any $x \in \Sigma, n \geq 1$ and $K \subset \varphi_{\underline{x}(n)}(I \times \mathbb{R})$ we have

$$
\begin{aligned}
\pi_{*} \nu\left(\varphi_{x(n)}^{-1}(K)\right) & =\int_{K} \exp \left(-S_{n}\left(t f_{W}+f_{H}\right)\right) d\left(\pi_{*} \nu\right) \\
& \simeq \pi_{*} \nu(K) \exp \left(-S_{n}\left(t f_{W}+f_{H}\right)(\underline{x})\right)
\end{aligned}
$$

In particular, for any $\underline{x} \in \Sigma$ and $n \geq 1$,

$$
\tilde{\pi}_{*} \nu\left(\psi_{\underline{x}(n)}(I)\right)=\pi_{*} \nu\left(\varphi_{\underline{x}(n)}(E)\right) \simeq \exp \left(S_{n}\left(t f_{W}+f_{H}\right)(\underline{x})\right)
$$

We now obtain a volume lemma for the measure $\pi_{*} \nu$ (compare to Lemma 8 of [PU])

LEMMA 9 For $\nu$ and $\mu$-almost all $x \in \Sigma$ we have

$$
\liminf _{n \rightarrow \infty} \frac{\log \left(\pi_{*} \nu(K(\underline{x}, n))\right)}{\log |\tilde{\pi}(\underline{x}(n))|}=H D\left(\pi_{*} \nu\right)
$$

Proof Let

$$
L(\underline{x})=\liminf _{n \rightarrow \infty} \frac{\log \left(\pi_{*} \nu(\underline{\kappa}(\underline{x}, n))\right)}{\log |\tilde{\pi}(\underline{x}(n))|}
$$

If $\underline{y}=\sigma(\underline{x})$ then $K(\underline{y}, n-1) \supset \varphi_{x_{1}}^{-1}(K(\underline{x}, n))$ and we get by $(32)$ that $\pi_{*} \nu(K(\underline{y}, n-$ $1)) \geq \pi_{*} \nu(K(\underline{x}, n))$ Hence

$$
\frac{\log \pi_{*} \nu(K(y, n-1))}{\log |\tilde{\pi} \underline{y}(n-1)|} \leq \frac{\log \pi_{*} \nu(K(\underline{x}, n))}{\log |\tilde{\pi} \underline{y}(n-1)|} \leq \frac{\log \pi_{*} \nu(K(\underline{x}, n))}{\log |\tilde{\pi} \underline{x}(n)|-\log a}
$$

Lettıng $n \rightarrow \infty$ we get $L(\sigma \underline{x}) \leq L(\underline{x})$ As $\sigma \quad \Sigma \rightarrow \Sigma$ is ergodic with respect to $\mu$, this implies that $L(\underline{x})$ is constant $\mu$-almost everywhere and equivalently $\nu$-almost everywhere We denote this $\mu$-almost sure value by $L$ Since $B(\pi(\underline{x}), 2|\tilde{\pi}(\underline{x}(n))|) \supset$ $K(\underline{x}, n)$, we obtain

$$
\lim _{n \rightarrow \infty} \frac{\log \pi_{*} \nu B(\pi(\underline{x}), 2|\tilde{\pi}(\underline{x}(n))|)}{\log |\tilde{\pi}(\underline{x}(n))|} \leq L(\underline{x})
$$

The Frostman Lemma (stated in the introduction) now gives $H D(\pi, \nu) \leq L$

The calculation of the other inequality is more complicated The main technical problem we face in obtaining the lower bound on $H D\left(\pi_{*} \nu\right)$ is that we do not have 
a good description of the density of $\pi_{*} \nu$ around points of $E$ with more than one corresponding symbol sequence We now make some estımates which will enable us to deal with this problem

First note that, since $P\left(t f_{W}+f_{H}\right)=0$, by the definition of pressure we have

$$
\limsup _{n \rightarrow \infty} \frac{1}{n} \log \sum_{\underline{x} \in B_{n}} \exp \left(S_{n}\left(t f_{W}+f_{H}\right)(\underline{x})\right)=0
$$

where for each $n \geq 1, B_{n} \subset \Sigma$ is a maximal set such that if $x, y \in B_{n}$ and $\underline{x} \neq \underline{y}$ then $\underline{x}(n) \neq y(n)$ By (3 3) this is equivalent to the formula

$$
\limsup _{n \rightarrow \infty} \frac{1}{n} \log \sum_{\underline{x}(n)} \tilde{\pi}_{*} \nu(\tilde{\pi}(\underline{x}(n)))=0
$$

Thus, given $\varepsilon>0$, for large enough $n$ we have $\sum_{\underline{x}(n)} \tilde{\pi}_{*} \nu(\tilde{\pi}(\underline{x}(n))) \leq e^{\frac{1}{2} \varepsilon n}$ In particular

$$
\sum_{n=1}^{\infty} \sum_{\underline{x}(n)} e^{-\varepsilon n} \tilde{\pi}_{*} \nu(\tilde{\pi}(\underline{x}(n)))<\infty
$$

Now fix $\beta>0$, choose some $n \geq 1$ and define $m=1+[(1+\beta) n]$ Corresponding to each interval $\tilde{\pi}(\underline{x}(n))$ there are two endpoints $x^{1}$ and $x^{2}$ and two sequences such that $\tilde{\pi}\left(\underline{x}^{1}\right)=x^{1}, \tilde{\pi}\left(\underline{x}^{2}\right)=x^{2}$, and $\underline{x}^{1}(n)=\underline{x}^{2}(n)=\underline{x}(n)$ Put

$$
A(\beta, n)=\bigcup_{\underline{x}(n)}\left(\tilde{\pi}\left(\underline{x}^{1}(m)\right) \cup \tilde{\pi}\left(\underline{x}^{2}(m)\right)\right)
$$

We can now use $\left(\begin{array}{ll}3 & 3)\end{array}\right)$ to estımate $\tilde{\pi}_{*} \nu(A(\beta, n))$,

$$
\begin{aligned}
\tilde{\pi}_{*} \nu(A(\beta, n)) & =\sum_{\underline{x}(n)}\left(\tilde{\pi}_{*} \nu\left(\tilde{\pi}\left(\underline{x}^{1}(m)\right)\right)+\tilde{\pi}_{*} \nu\left(\tilde{\pi}\left(\underline{x}^{2}(m)\right)\right)\right) \\
& \simeq \sum_{\underline{x}(n)}\left(\exp \left(S_{m}\left(t f_{W}+f_{H}\right)\left(\underline{x}^{1}\right)\right)+\exp \left(S_{m}\left(t f_{W}+f_{H}\right)\left(\underline{x}^{2}\right)\right)\right) \\
& \leq \sum_{\underline{x}(n)}\left(\exp \left(S_{n}\left(t f_{W}+f_{H}\right)\left(\underline{x}^{1}\right)\right)\right. \\
& \left.+\exp \left(S_{n}\left(t f_{W}+f_{H}\right)\left(\underline{x}^{2}\right)\right)\right) \exp ((t \log A+\log B) \beta n) \\
& \simeq 2 \exp ((t \log A+\log B) \beta n) \sum_{\underline{x}(n)} \tilde{\pi}_{*} \nu(\tilde{\pi}(\underline{x}(n)))
\end{aligned}
$$

Using the fact that $\log A, \log B<0,(34)$ implies that

$$
\sum_{n=1}^{\infty} \tilde{\pi}_{*} \nu(A(\beta, n))<\infty
$$

Now observe that by Lemma 3 we have

$$
\begin{aligned}
\left|\tilde{\pi}\left(\underline{x}^{1}(m)\right)\right| & \simeq \exp \left(S_{m} f_{W}\left(\underline{x}^{1}\right)\right) \\
& \geq \exp \left(S_{n} f_{W}\left(\underline{x}^{1}\right)\right) \exp (\beta n \log a) \\
& \simeq \exp (\beta n \log a)\left|\tilde{\pi}\left(\underline{x}^{1}(n)\right)\right|
\end{aligned}
$$

The same estimate is obviously true for $\left|\tilde{\pi}\left(\underline{x}^{2}(m)\right)\right|$ Thus if $\underline{x}$ is not in $\tilde{\pi}^{-1}(A(\beta, n))$ then

$$
K(\underline{x}, n) \supset B\left(\pi(\underline{x}), c a^{\beta n}|\tilde{\pi}(\underline{x}(n))|\right),
$$


where $0<c \leq 1$ is a universal constant Now by (3 5) and the Borel-Cantelli lemma, $\nu$-almost every $\underline{x} \in \Sigma$ does not belong to $\tilde{\pi}^{-1}(A(\beta, n))$ for all large $n$ Therefore

$$
\begin{aligned}
L & \leq \liminf _{n \rightarrow \infty} \frac{\log \tilde{\pi}_{*} \nu B\left(\pi(\underline{x}), c a^{\beta n}|\tilde{\pi}(\underline{x}(n))|\right)}{\log |\tilde{\pi}(\underline{x}(n))|} \\
& =\liminf _{n \rightarrow \infty} \frac{\log c+\beta n \log a+\log |\tilde{\pi}(\underline{x}(n))|}{\log |\tilde{\pi}(\underline{x}(n))|} \frac{\log \tilde{\pi}_{*} \nu B\left(\pi(\underline{x}), c a^{\beta n}|\tilde{\pi}(\underline{x}(n))|\right)}{\log \left(c a^{\beta n}|\tilde{\pi}(\underline{x}(n))|\right)}
\end{aligned}
$$

and as

$$
1 \leq \frac{\log c+\beta n \log a+\log |\tilde{\pi}(\underline{x}(n))|}{\log |\tilde{\pi}(\underline{x}(n))|} \leq 1+\beta \frac{\log a}{\log A}+\frac{1}{n} \frac{\log c}{\log A}
$$

we deduce from Frostman's lemma that

$$
L \leq\left(1+\beta \frac{\log a}{\log A}\right) H D\left(\pi_{*} \nu\right)
$$

Lettıng $\beta \rightarrow 0$ we get $L \leq H D\left(\pi_{*} \nu\right)$ which proves the lemma

We shall obtain a formula for the dimension of $\pi_{*} \nu$ in terms of (amongst other things) the dimension of the measure $\left(p_{H} \pi\right)_{*} \nu$ The following two lemmas are volume lemmas for $\left(p_{H} \pi\right)_{*} \nu$

LEMMA 10 For $\mu$ and $\nu$-almost every $x \in \Sigma$ we have

$$
\liminf _{r \rightarrow 0} \frac{\log \left(p_{H} \pi\right)_{*} \nu\left(B\left(p_{H} \pi(x), r\right)\right)}{\log r}=H D\left(\left(p_{H} \pi\right)_{*} \nu\right)
$$

Proof Denote the map $p_{H} \pi$ by $\pi_{H}$ and the measure $\left(p_{H} \pi\right)_{*} \nu$ by $\bar{\nu}$ For every $\underline{x} \in \Sigma$ let

$$
L(\underline{x})=\liminf _{r \rightarrow 0} \frac{\log \bar{\nu}\left(B\left(\pi_{H}(\underline{x}), r\right)\right)}{\log t}
$$

By Frostman's lemma it is sufficient to show that $L(\underline{x})$ is $\nu$-almost surely constant Now for every $n \geq 1$ and $x \in \Sigma$ we have

$$
\varphi_{x_{1}}^{-1}\left(\tilde{\pi}(\underline{x}(1)) \times B\left(\pi_{H}(\underline{x}), r\right)\right) \supset I \times B\left(\pi_{H}(\sigma \underline{x}), B^{-1} r\right) \supset I \times B\left(\pi_{H}(\sigma \underline{x}), r\right)
$$

Thus, puttıng $D=\max \left(1, \sup \left\{\exp \left(-t f_{W}-f_{H}\right)(\underline{x}) \underline{x} \in \Sigma\right\}\right)$ we obtain

$$
\begin{aligned}
\bar{\nu}\left(B\left(\pi_{H}(\sigma \underline{x}), r\right)\right) & =\pi_{*} \nu\left(I \times B\left(\pi_{H}(\sigma \underline{x}), r\right)\right) \\
& \leq \pi_{*} \nu\left(\varphi_{r_{1}}^{-1}\left(\tilde{\pi}(\underline{x}(1)) \times B\left(\pi_{H}(\underline{x}), r\right)\right)\right) \\
& \leq D \pi_{*} \nu\left(\left(\tilde{\pi}(\underline{x}(1)) \times B\left(\pi_{H}(\underline{x}), r\right)\right)\right) \\
& \leq D \pi_{*} \nu\left(I \times B\left(\pi_{H}(\underline{x}), r\right)\right) \\
& =D \bar{\nu}\left(B\left(\pi_{H}(\underline{x}), r\right)\right)
\end{aligned}
$$

Consequently, for every $0<\mathrm{r}<1$ we get

$$
\frac{\log \bar{\nu}\left(B\left(\pi_{H}(\sigma \underline{x}), r\right)\right)}{\log r} \geq \frac{\log \bar{\nu}\left(B\left(\pi_{H}(\underline{x}), r\right)\right)}{\log r}+\frac{\log D}{\log r}
$$

Thus, letting $r \rightarrow 0$, we obtain $L(\sigma \underline{x}) \geq L(\underline{x})$ Since

$$
\int L(\sigma \underline{x}) d \mu(\underline{x})=\int L(\underline{x}) d \mu(\underline{x})
$$


this implies that $L(\sigma \underline{x})=L(\underline{x})$ for $\mu$-a e $x \in \Sigma$ Therefore, by ergodicity of $\mu$ with respect to $\sigma, L(\underline{x})$ is $\mu$-a s constant and hence also $\nu$-a s constant (as $\mu$ and $\nu$ are equivalent)

The next lemma says that the limit around the point $p_{H} \pi \underline{x}$ in the statement of Lemma 10 can be replaced by a limit taken along a sequence of points $p_{H} \pi \sigma^{n} \underline{x}$

LEMMA 11 If $\theta_{n} \Sigma \rightarrow(0, \infty)$ is a sequence of measurable functions such that

$$
\lim _{n \rightarrow \infty} \frac{1}{n} \log \theta_{n}(\underline{x})=\theta
$$

for $\mu$ a a $\underline{x} \in \Sigma$ and some $\theta<0$, then

$$
\lim _{n \rightarrow \infty} \frac{\log \left(p_{H} \pi\right)_{*} \nu\left(B\left(p_{H} \pi\left(\sigma^{n} \underline{x}\right), \theta_{n}(\underline{x})\right)\right)}{\log \theta_{n}(\underline{x})}=H D\left(\left(p_{H} \pi\right)_{*} \nu\right),
$$

for $\mu$ a a $\underline{x} \in \Sigma$

Proof Define $\pi_{H}$ and $\bar{\nu}$ as in the last lemma For every $\underline{x} \in \Sigma$ let

$$
L(\underline{x})=\liminf _{n \rightarrow \infty} \frac{\log \bar{\nu}\left(B\left(\pi_{H}\left(\sigma^{n} \underline{x}\right), \theta^{n}(\underline{x})\right)\right)}{\log \theta_{n}(\underline{x})}
$$

First we show that

$$
L(\underline{x}) \geq H D(\bar{\nu}) \text { for } \mu \text { a e } \underline{x} \in \Sigma
$$

Let $Z=\left\{\underline{x} \in \Sigma \lim _{n \rightarrow \infty} n^{-1} \log \theta_{n}(\underline{x})=\theta\right\}$ By assumption $Z$ has $\mu$-measure 1 From the last lemma and Egorov's Theorem it follows that for every $p<H D(\bar{\nu})$ there exists $r(p)>0$ and a Borel set $S \subset \Sigma$ such that $\mu(S)>0$ and

$$
\frac{\log \bar{\nu}\left(B\left(\pi_{H}(\underline{x}), r\right)\right)}{\log r} \geq p
$$

for every $\underline{x} \in S$ and $0<r \leq r(p)$ By ergodicity of $\mu$, for $\mu$-almost all $\underline{x} \in \Sigma$ there is an increasing sequence $\left\{n_{j}\right\}$ (depending on $\underline{x}$ ) such that $\sigma^{n},(\underline{x}) \in S$ for $j=1,2$, Now take $J_{0} \geq 1$ (again depending on $\underline{x}$ ) large enough that $\theta_{n}(\underline{x}) \leq r(p)$ for every $n \geq n_{j_{0}}$ Then by (38) we have

$$
\frac{\log \bar{\nu}\left(B\left(\pi_{H}\left(\sigma^{n_{j}} \underline{x}\right), \theta_{n_{1}}(\underline{x})\right)\right)}{\log \theta_{n_{i}}(\underline{x})} \geq p
$$

for every $J \geq J_{0}$ Fix now $0<\varepsilon<-\theta$ and let $l_{-1} \geq 1$ be so large that

$$
\theta-\varepsilon \leq \frac{1}{m} \log \theta_{m}(\underline{x}) \leq \theta+\varepsilon \text { for every } m \geq l_{-1}
$$

Let $t(m)=[(\theta+\varepsilon) /(\theta-\varepsilon) m] \leq m$ and let $J(m)$ be the largest integer such that $n_{j(m)} \leq t(m)$ Since $\lim _{j \rightarrow \infty} n_{j}=\infty$, there exists $l_{0} \geq l_{-1}$ so large that $n_{j(m)} \geq l_{-1}$ and $J(m) \geq J_{0}$ for $m \geq l_{0}$ Using (310) we therefore have that for every $m \geq l_{0}$,

$$
\log \theta_{m}(\underline{x}) \leq(\theta+\varepsilon) m \leq(\theta-\varepsilon) t(m) \leq(\theta-\varepsilon) n_{j(m)} \leq \log \theta_{n_{,(m)}}(\underline{x})
$$


Hence $\theta_{m}(\underline{x}) \leq \theta_{n, t m)}(\underline{x})$ and using (3 6) we get for $m \geq l_{0}$ that

$$
\begin{aligned}
\bar{\nu}\left(B\left(\pi_{H}\left(\sigma^{m} \underline{x}\right), \theta_{m}(\underline{x})\right)\right) & \leq D^{m-n_{\gamma^{(m)}}} \bar{\nu}\left(B\left(\pi_{H}\left(\sigma^{n_{\lambda^{(m)}}} \underline{x}\right), \theta_{m}(\underline{x})\right)\right) \\
& \leq D^{m-n_{\left.\gamma^{(m)}\right)}} \bar{\nu}\left(B\left(\pi_{H}\left(\sigma^{n_{\gamma^{(m)}}} \underline{x}\right), \theta_{n_{f^{(m)}}}(\underline{x})\right)\right)
\end{aligned}
$$

Therefore for $m \geq l_{0}$ we have,

$$
\begin{aligned}
& \frac{\log \bar{\nu}\left(B\left(\pi_{H}\left(\sigma^{m} \underline{x}\right), \theta_{m}(\underline{x})\right)\right)}{\log \theta_{m}(\underline{x})} \\
& \geq \frac{\left(m-n_{f(m)}\right) \log D}{\log \theta_{m}(\underline{x})}+\frac{\log \theta_{n_{l(m)}}(\underline{x})}{\log \theta_{m}(\underline{x})} \frac{\log \bar{\nu}\left(B\left(\pi_{H}\left(\sigma^{n_{J(m)}} \underline{x}\right), \theta_{n_{f(m)}}(\underline{x})\right)\right)}{\log \theta_{n_{f(m)}}(\underline{x})} \\
& =\frac{\frac{\left(m-n_{j(m)}\right)}{m} \log D}{\frac{1}{m} \log \theta_{m}(\underline{x})}+\frac{n_{J(m)}}{m} \frac{\frac{1}{n_{J(m)}} \log \theta_{n_{l(m)}}(\underline{x})}{\frac{1}{m} \log \theta_{m}(\underline{x})} \frac{\log \bar{\nu}\left(B\left(\pi_{H}\left(\sigma^{n_{f(m)}} \underline{x}\right), \theta_{n_{j(m)}}(\underline{x})\right)\right)}{\log \theta_{n_{(m)}(\underline{x})}}
\end{aligned}
$$

By the definition of $t(m)$ and $J(m)$ we have $n_{f(m)+1} \geq\{(\theta+\varepsilon) /(\theta-\varepsilon)\} m$ From the Birkhoff ergodic theorem we deduce that $\lim _{j \rightarrow \infty}\left(n_{j} / n_{j+1}\right)=1$ Therefore for $m \geq l_{0}$ large enough we have

$$
n_{J(m)} \geq(1-\varepsilon) n_{J(m)+1} \geq(1-\varepsilon) \frac{\theta+\varepsilon}{\theta-\varepsilon} m
$$

Thus taking the limit of $m \rightarrow \infty$ from (3 11) and (3 9) we get,

$$
L(\underline{x}) \geq \frac{\varepsilon \frac{\theta+\varepsilon}{\theta-\varepsilon} \log D}{\theta}+(1-\varepsilon) \frac{\theta+\varepsilon}{\theta-\varepsilon} \frac{\theta}{\theta} p
$$

Lettıng $\varepsilon \succ 0$ we get $L(\underline{x}) \geq p$ In particular, as $\mu(Z)=1$ we get $L(\underline{x}) \geq p$ for $\mu$-a a $\underline{x} \in \Sigma$ Letting $p \nearrow H D(\bar{\nu})$ we obtain inequality (37)

In order to prove the converse inequality, let

$$
R=\sup \operatorname{ess}(L)=\inf \{\sup \{L(\underline{x}) \underline{x} \in \Sigma\} \mu(X)=1\}
$$

From Egorov's theorem we have that for every $Q<R$ there exists an integer $l_{1} \geq 1$ and a Borel set $Y_{1} \subset \Sigma$ such that $\mu\left(Y_{1}\right)>0$ and

$$
\frac{\log \bar{\nu} B\left(\pi_{H}\left(\sigma^{n} \underline{x}\right), \theta_{n}(\underline{x})\right)}{\log \theta_{n}(\underline{x})} \geq Q \text { for every } \underline{x} \in Y_{1} \text { and } n \geq l_{1}
$$

Also by the same theorem, for every $0<\varepsilon<-\theta$ there exists an integer $l_{2} \geq 1$ and a Borel set $Y_{2} \subset \Sigma$ such that $\mu\left(Y_{2}\right) \geq 1-\frac{1}{2} \mu\left(Y_{1}\right)$ and

$$
(\theta-\varepsilon) n \leq \log \theta_{n}(\underline{x}) \leq(\theta+\varepsilon) n \text { for every } \underline{x} \in Y_{2} \text { and } n \geq l_{2}
$$

Now, for any $\underline{x} \in \Sigma$ let $m_{k}=m_{k}(\underline{x}), k=1,2$, be the increasing sequence of integers $m \geq 0$ for which $\sigma^{-m}(\underline{x}) \cap Y_{1} \cap Y_{2} \neq \varnothing$ As $\mu\left(Y_{1} \cap Y_{2}\right) \geq \frac{1}{2} \mu\left(Y_{1}\right)>0$ we deduce from the Birkhoff ergodic theorem applied to the Rochlın natural extension of the system 
$(\sigma, \mu)$ that for $\mu$-a e $\underline{x} \in \Sigma$, say $\underline{x} \in V$ with $\mu(V)=1$, the sequence $m_{k}=m_{k}(\underline{x})$ is infinite and furthermore that

$$
\lim _{k \rightarrow \infty} \frac{m_{k}}{m_{k+1}}=1
$$

Consider now any $\underline{x} \in V$, let $m_{h}=m_{k}(\underline{x})$ and let $\left\{\underline{x}_{k}\right\}_{k=1}^{\infty}$ be a sequence consistıng of elements of $Y_{1} \cap Y_{2}$ such that $\sigma^{m_{k}}\left(\underline{x}_{k}\right)=\underline{x}$ for every $k=1,2, \quad$ For every small enough $0<r<1$ let $p=p(r) \geq 1$ be the largest integer such that

$$
\theta_{m_{p}}\left(\underline{x}_{p}\right) \geq r
$$

so that in particular,

$$
\theta_{m_{p+1}}\left(\underline{x}_{p+1}\right)<r
$$

(a largest such $p$ exists because ( 313 ) holds for each $\underline{x}_{k}$ ) Thus $p(r) \rightarrow \infty$ as $r \rightarrow 0$ and so there exists $0<r_{0}<1$ such that

$$
m_{p(r)} \geq \max \left(l_{1}, l_{2}\right) \text { for every } 0<r \leq r_{0}
$$

Thus for any $r \in\left(0, r_{0}\right]$ it follows from (3 15) that $B\left(\pi_{H}(\underline{x}), r\right) \subset B\left(\pi_{H}(\underline{x}), \theta_{m_{p}}\left(\underline{x}_{p}\right)\right)$ From (3 12) we therefore get

$$
\begin{aligned}
\frac{\log \bar{\nu} B\left(\pi_{H}(\underline{x}), r\right)}{\log r} & \geq \frac{\log \bar{\nu} B\left(\pi_{H}(\underline{x}), \theta_{m_{p}}\left(\underline{x}_{p}\right)\right)}{\log r} \\
& =\frac{\log \bar{\nu} B\left(\pi_{H} \sigma^{m_{p}}\left(\underline{x}_{p}\right), \theta_{m}\left(\underline{x}_{p}\right)\right)}{\log \theta_{m_{p}}\left(\underline{x}_{p}\right)} \frac{\log \theta_{m_{p}}\left(\underline{x}_{p}\right)}{\log r} \\
& \geq Q \frac{\log \theta_{m_{p}}\left(\underline{x}_{p}\right)}{\log r}
\end{aligned}
$$

In view of (3 13) and (3 17) we obtain $\log \theta_{m_{p}}\left(\underline{x}_{p}\right) \leq(\theta+\varepsilon) m_{p}$ and $\log \theta_{m_{p+1}}\left(\underline{x}_{p+1}\right) \geq$ $(\theta-\varepsilon) m_{p+1}$ Consequently

$$
\frac{\log \theta_{m_{r}}\left(\underline{x}_{p}\right)}{\log \theta_{m_{p+1}}\left(\underline{x}_{p+1}\right)} \geq \frac{\theta+\varepsilon}{\theta-\varepsilon} \frac{m_{p}}{m_{p+1}}
$$

From this, (3 16) and (3 18) we get

$$
\frac{\log \bar{\nu} B\left(\pi_{H}(\underline{x}), r\right)}{\log r} \geq Q \frac{\theta+\varepsilon}{\theta-\varepsilon} \frac{m_{p}}{m_{p+1}}
$$

Hence, lettıng $r \rightarrow 0$, by Lemma 10, and (3 14) we obtain $H D(\bar{\nu}) \geq Q[(\theta+\varepsilon) /(\theta-\varepsilon)]$, and lettıng first $\varepsilon \rightarrow 0$ and then $Q \nearrow R$ we get $H D(\bar{\nu}) \geq R$ This completes the proof

We shall now prove the main result of the paper (compare with Theorem 7 of [PU])

THEOREM 12 The Hausdorff dimension of $\pi_{*} \mu$ satısfies

$$
H D\left(\pi_{*} \mu\right)=t+\frac{\chi_{H}}{\chi_{H}} H D\left(\left(p_{H} \pi\right)_{*} \mu\right)\left(1-\frac{\chi_{H}}{\chi_{W}}\right)
$$

where $\chi_{W}=\int f_{\mu} d \mu$ and $\chi_{H}=\int f_{H} d \mu$ 
Proof As $\mu$ and $\nu$ are equivalent, $H D\left(\pi_{*} \mu\right)=H D\left(\pi_{*} \nu\right)$ and it is enough to prove that

$$
H D\left(\pi_{*} \nu\right)=t+\frac{\chi_{H}}{\chi_{W}}+H D\left(\left(p_{H} \pi\right)_{*} \mu\right)\left(1-\frac{\chi_{H}}{\chi_{W}}\right)
$$

We keep the notation $\pi_{H}=p_{H} \circ \pi$, and $\bar{\nu}=\left(p_{H} \pi\right)_{*} \nu$, and we put $\bar{\mu}=\left(p_{H} \pi\right)_{*} \mu$ Recall from Lemma 7 that $K(\underline{x}, n)$ is a square of side length $|\tilde{\pi}(\underline{x}(n))|$ about $\pi(\underline{x})$, then by Lemma 7 there exists a constant $0<C \leq 1$ such that

$$
\begin{aligned}
& B\left(p_{H} \pi\left(\sigma^{n} \underline{x}\right), C|\tilde{\pi}(\underline{x}(n))| \exp \left(-S_{n} f_{H}(\underline{x})\right)\right) \subset p_{H} \varphi_{\underline{x}(n)}^{-1} K(\underline{x}, n) \\
& \quad \subset B\left(p_{H} \pi\left(\sigma^{n} \underline{x}\right), C^{-1}|\tilde{\pi}(\underline{x}(n))| \exp \left(-S_{n} f_{H}(\underline{x})\right)\right)
\end{aligned}
$$

for every $\underline{x} \in \Sigma$ and $n \geq 1$ In view of Lemma 8 ,

$$
\pi_{*} \nu(K(\underline{x}, n)) \simeq \exp \left(S_{n}\left(t f_{W}+f_{H}\right)(\underline{x})\right) \pi_{*} \nu\left(\varphi_{\underline{x}(n)}^{-1} K(\underline{x}, n)\right)
$$

Take now

$$
\theta_{n}^{1}(\underline{x})=C|\tilde{\pi}(\underline{x}(n))| \exp \left(-S_{n} f_{H}(\underline{x})\right)
$$

and

$$
\theta_{n}^{(2)}(\underline{x})=C^{-1} \cap \tilde{\pi}(\underline{x}(n)) \mid \exp \left(-S_{n} f_{H}(\underline{x})\right)
$$

for $\underline{x} \in \Sigma$ and $n=1,2, \quad$ As $\varphi_{\underline{x}(n)}^{-1}(K(\underline{x}, n))$ is a rectangle which lies across the full width of the unit interval, by (3 19) and (3 20) we get

$$
\begin{aligned}
\log \pi_{*} \nu(K(\underline{x}, n)) & =S_{n}\left(t f_{W}+f_{H}\right)(\underline{x})+\log \pi_{*} \nu\left(\varphi_{\underline{x}(n)}^{-1} K(\underline{x}, n)\right)+X_{1} \\
& \geq S_{n}\left(t f_{W}+f_{H}\right)(\underline{x})+\log \bar{\nu}\left(B\left(\pi_{H}\left(\sigma^{n} \underline{x}\right), \theta_{n}^{(1)}(\underline{x})\right)+X_{1}\right.
\end{aligned}
$$

and

$$
\log \pi_{*} \nu(K(\underline{x}, n)) \leq S_{n}\left(t f_{W}+f_{H}\right)(\underline{x})+\log \bar{\nu}\left(B\left(\pi_{H}\left(\sigma^{n} \underline{x}\right), \theta_{n}^{(2)}(\underline{x})\right)+X_{2},\right.
$$

where $X_{1}, X_{2}>0$ are uniformly bounded, both from above and from zero, with respect to $\underline{x} \in \Sigma$ and $n \geq 1$ Therefore

$\frac{\log \pi_{*} \nu(K(\underline{x}, n))}{\log |\tilde{\pi}(x(n))|}$

$$
\leq \frac{S_{n}\left(t f_{W}+f_{H}\right)(\underline{x})}{\log |\tilde{\pi}(\underline{x}(n))|}+\frac{\log \theta_{n}^{(1)}(\underline{x})}{\log |\tilde{\pi}(\underline{x}(n))|} \frac{\log \bar{\nu}\left(B\left(\pi_{H}\left(\sigma^{n} \underline{x}\right), \theta_{n}^{(1)}(\underline{x})\right)\right)}{\log \theta_{n}^{(1)}(\underline{x})}+\frac{\log X_{1}}{\log |\tilde{\pi}(\underline{x}(n))|}
$$

and

$$
\begin{aligned}
& \frac{\log \pi_{*} \nu(K(\underline{x}, n))}{\log |\tilde{\pi}(\underline{x}(n))|} \\
& \quad \geq \frac{S_{n}\left(t f_{W}+f_{H}\right)(\underline{x})}{\log |\tilde{\pi}(\underline{x}(n))|}+\frac{\log \theta_{n}^{(2)}(\underline{x})}{\log |\tilde{\pi}(\underline{x}(n))|} \frac{\log \bar{\nu}\left(B\left(\pi_{H}\left(\sigma^{n} \underline{x}\right), \theta_{n}^{(2)}(x)\right)\right.}{\log \theta_{n}^{(2)}(\underline{x})}+\frac{\log X_{2}}{\log |\tilde{\pi}(\underline{x}(n))|}
\end{aligned}
$$

Now, by Lemma 3 and the Birkhoff ergodic theorem we know that for $\mu$-a a $x \in \Sigma$,

$$
\lim _{n \rightarrow \infty} \frac{1}{n} \log \theta_{n}^{(1)}(\underline{x})=\lim _{n \rightarrow \infty} \frac{1}{n} \log \theta_{n}^{(2)}(\underline{x})=\int f_{W}-f_{H} d \mu<0
$$


This property means that we can apply Lemma 11 to each of the sequences $\left\{\theta_{n}^{(1)}\right\}$ and $\left\{\theta_{n}^{(2)}\right\}$ Using this Lemma, taking the limit of $n \rightarrow \infty$ in (3 21) and (3 22) gives

$$
H D\left(\pi_{*} \nu\right) \leq \frac{t \chi_{W}+\chi_{H}}{\chi_{W}}+H D(\bar{\nu}) \frac{\chi_{W}-\chi_{H}}{\chi_{W}}
$$

and

$$
H D\left(\pi_{*} \nu\right) \geq \frac{t \chi_{W}+\chi_{H}}{\chi_{W}}+H D(\bar{\nu}) \frac{\chi_{W}-\chi_{H}}{\chi_{W}}
$$

Rearranging these expressions gives the claimed dimension formula The proof is thus complete

As $\mu$ is an atom free measure, the set $E\left(\bmod \pi_{*} \mu\right)$ can be regarded as the graph of a Borel function $f I \rightarrow \mathbb{B}$ defined $\tilde{\pi}_{*} \mu$ almost everywhere In this context the measure $\left(p_{H} \pi\right)_{*} \mu$ can be interpreted as the probability distribution of the random variable $f I \rightarrow \mathbb{R}$ defined on the probability space $\left(I, \tilde{\pi}_{*} \mu\right)$ The following theorem gives a positive answer to one direction of the conjecture stated in the introduction

THEOREM 13 If $E$ has a weak invariant foliation $(*)$ and $H D\left(\left(p_{H} \pi\right)_{*} \mu\right)=1$ then $H D(E)=D_{B}(E)=t+1$

Proof From the remark after Theorem 6 we have $D_{B}(E) \leq t+1$, and Theorem 12 gives $H D(E) \geq H D\left(\pi_{*} \mu\right)=t+1$ As $H D(E) \leq D_{B}(E)$, this finishes the proof

Remark. In the above theorem we do not use the assumption that $E$ satisfies the Darboux property

We can also give a partial positive answer in the second direction of the conjecture stated in the introduction

THEOREM 14 Suppose that $E$ has a weak invariant foliation (*) and that $H D\left(\left(p_{H} \pi\right)_{*} \mu\right)<1$ Then $H_{t+1}(E)=0$ where $H_{t+1}(E)$ is the $(t+1)$-dimensional Hausdorff measure of $E$

Proof Theorem 12 implies the existence of a Borel set $F \subset E$ such that $\pi_{*} \mu(F)=1$ and $H D(F)<t+1$ Hence, if $Z=E \backslash F$ then $\tilde{\pi}_{*} \mu\left(p_{W}(Z)\right)=0$ Since the sequence $\left\{\{\tilde{\pi}(\underline{x}(n))\}_{\underline{x}}\right\}_{n=1}^{\infty}$ of partitions $\left(\bmod \pi_{*} \mu\right)$ of $I$ is increasing and generates the Borel $\sigma$-algebra on $I$, for given $\theta, \gamma>0$ we can find a countable subset $\left\{\underline{x}^{\prime}\right\}_{j=1}^{\infty}$ of $\Sigma$ and a sequence $\left\{n_{j}\right\}_{j=1}^{\infty}$ of positive integers such that

$$
p_{W}(Z) \subset \bigcup_{j=1}^{\infty} \tilde{\pi}\left(\underline{x}^{\jmath}\left(n_{j}\right)\right), \quad \sum_{j=1}^{\infty} \tilde{\pi}_{*} \nu\left(\tilde{\pi}\left(\underline{x}^{\jmath}\left(n_{j}\right)\right)\right) \leq \gamma
$$

and

$$
\operatorname{diam}\left(\tilde{\pi}\left(\underline{x}^{j}\left(n_{j}\right)\right)\right) \leq \theta \text { for every } J \geq 1
$$

By Lemmas 3 and 4 , every set $\varphi_{x^{\prime}(n,)}(E)$ can be covered by at most const $\exp \left(S_{n_{j}}\left(f_{H}-\right.\right.$ $\left.\left.f_{W}\right)\left(\underline{x}^{j}\right)\right)$ squares with edges of length $\left|\tilde{\pi}\left(\underline{x}^{j}\left(n_{j}\right)\right)\right|$ We can therefore estimate Hausdorff measure as follows Let

$$
H_{t+1}(Z, \theta)=\operatorname{lnf}\left\{\sum_{j=1}^{\infty}\left(\operatorname{diam} U_{j}\right)^{t+1} \bigcup_{J} U_{J} \supset Z, \operatorname{diam} U_{J} \leq \theta, U_{j} \text { open }\right\}
$$


Then

$$
\begin{aligned}
H_{t+1}(Z, \theta) & \leq \text { const } \sum_{j=1}^{\infty} \exp \left(S_{n_{j}}\left(f_{H}-f_{W}\right)\left(\underline{x}^{j}\right)\right)\left(\exp \left(S_{n_{j}} f_{W}\left(\underline{x}^{j}\right)\right)\right)^{t+1} \\
& =\text { const } \sum_{j=1}^{\infty} \exp \left(S_{n_{j}}\left(t f_{W}+f_{H}\right)\left(\underline{x}^{j}\right)\right)
\end{aligned}
$$

Now as $P\left(t f_{W}+f_{H}\right)=0$ it follows from (29) that

$$
H_{t+1}(Z, \theta) \leq \mathrm{const} \sum_{j=1}^{\infty} \tilde{\pi}_{*} \nu\left(\tilde{\pi}\left(\underline{x}^{j}\left(n_{\jmath}\right)\right)\right) \leq \mathrm{const} \gamma
$$

If we now let $\gamma$ and then $\theta$ go to zero we get $H_{t+1}(Z)=0$ As $H_{t+1}(F)=0$ this gives $H_{t+1}(E)=p$

REMARK All the results of this section rely heavily on the assumption that $E$ has a weak invariant foliation, and we suspect that without it they are not true We shall develop this remark in a forthcoming paper

\section{Some examples of self-affine sets}

In this last section we describe some examples of self-affine sets for which we can check whether or not $H D\left(\left(p_{H} \pi\right)_{*} \mu\right)=1$ holds We begin with the following

PROPOSITION 15 Let $J$ be the convex hull of $p_{H}(E)$ If $E$ has a weak invariant foliation (*) and $J=\bigcup_{1} \tau_{1}(J)$ then $E$ satisfies the Darboux property

Proof By condition (*), the set $p_{H}(E)$ is self-similar under the maps $\tau_{\text {, Since } J \text { is }}$ compact and non-empty, the unıqueness of self-sımılar sets (see [H]) implies that $J=p_{H}(E)$ Now for any $x \in \Sigma$ and $n \geq 0$,

$$
p_{H} \varphi_{\underline{x}(n)}(E)=\tau_{\underline{x}(n)}(J)
$$

which is connected Hence $E$ satisfies the Darboux property

A simple family of self-affine sets can be defined as follows Take $0<p, \beta<1$ such that $\max (p, 1-p)<\beta$ and let $E(p, \beta)$ be the self-affine set determined by the contractions $\varphi_{0}, \varphi_{1} I \times \mathbb{R} \rightarrow I \times \mathbb{R}$ defined by

$$
\varphi_{0}(x, y)=(p x, \beta y), \quad \varphi_{1}(x, y)=(p+(1-p) x, \beta y+1-\beta)
$$

It is easy to check that $E(p, \beta)$ satisfies $(*)$ and that the assumptions of Proposition 15 are fulfilled in particular $E(p, \beta)$ satisfies the Darboux property The functions $f_{H}, f_{W} \Sigma \rightarrow \mathbb{R}$ associated to $E(p, \beta)$ are given by

$$
f_{H}(\underline{x}) \equiv \log \beta \quad f_{W}(\underline{x})= \begin{cases}\log p & \text { if } x_{1}=0 \\ \log (1-p) & \text { if } x_{1}=1\end{cases}
$$

The measure $\mu$ is particularly simple in this case Let $m$ be any $\sigma$-invariant probability measure on $\Sigma$ and let $q=m\left(\left\{\underline{x} \in \Sigma x_{1}=0\right\}\right)$ Then

$$
\begin{aligned}
& h_{m}(\sigma)+\int\left(s f_{W}+f_{H}\right) d m \\
& \quad \leq-q \log q-(1-q) \log (1-q)+s q \log p+s(1-q) \log (1-p)+\log \beta=F(s, q)
\end{aligned}
$$


and we have equality if and only if $\boldsymbol{m}$ is the product measure on $\Sigma$ determined by the probability vector $(q, 1-q)$ In order to find the corresponding number $t$ and the equilibrium state $\mu$ we have to find $t, q \in[0,1]$ such that $F(t, q)=0$ and $F(t, d) \leq$ $F(t, q)$ for every $d \in[0,1]$ With elementary calculus one obtains the following

PROPOSITION 16 The number $t$ is uniquely determined by the equation $p^{t}+(1-p)^{t}=$ $\beta^{-1}$ and the equilibrium state $\mu$ is the Bernoull measure given by the probabiltty vector $\left(p^{\prime} \beta,(1-p)^{t} \beta\right)$

Now observe that the map $p_{H} \pi \Sigma \rightarrow \mathbb{R}$ is given by

$$
p_{H} \pi(\underline{x})=(1-\beta) \sum_{n=1}^{\infty} \beta^{n-1} x_{n}
$$

This expresses $p_{H} \pi$ as a series of independent random variables on the probability space $(\Sigma, \mu)$ Therefore, the Fourier transform of the measure $\left(p_{H} \pi\right)_{*} \mu$ is the infinite product of the Fourier transforms of measures $\left((1-\beta) \beta^{n-1} x_{n}\right)_{*} \mu$ which can easily be computed by hand Using this one can prove, in the same way as in [E], the following

THEOREM 17 For all $p \in(0,1)$ there exists $\beta(p) \in(\max (p, 1-p), 1)$ and a set $Z(p)$ of full measure in $[\beta(p), 1)$ with the property that for any $\beta \in Z(p)$ the measure $\left(p_{H} \pi\right)_{*} \mu_{p \beta}$ is absolutely contınuous with respect to Lebesgue measure on $\mathbb{R}$ Furthermore $\lim _{\varepsilon \rightarrow 0} H D(([\beta(p), 1] \backslash Z(p)) \cap(1-\varepsilon, 1))=0$

Since the Hausdorff dimension of any measure that is absolutely continuous to Lebesgue measure on $\mathbb{R}$ is equal to 1 we can combine the above with Theorem 13 to get

CoRollary 18 For every $(p, \beta) \in \bigcup_{p \in(01)}\{p\} \times Z(p)$ we have $H D(E(p, \beta))=$ $D_{B}(E(p, \beta))$ In particular, the set of parameter values $(p, \beta)$ for which $H D(E(p, \beta))=D_{B}(E(p, \beta))$ has positive Lebesgue measure in $(0,1)^{2}$

The above class is a generalization of some examples considered in [PU] Define a mapping $h I \rightarrow I$ by

$$
h(x)= \begin{cases}p^{-1} x & 0 \leq x<p \\ (1-p)^{-1} x-p /(1-p) & p \leq x \leq 1\end{cases}
$$

and let $f I \rightarrow \mathbb{R}$ be the map $f(x)=(1-\beta) \sum_{n=0}^{\infty} \beta^{n} r_{n}(x)$, where $r_{n}=I_{[p 1]}{ }^{\circ} h^{n}$ It is easy to check that $E(p, \beta)$ coincides up to a countable set with the graph of $f$ Fixing $p=\frac{1}{2}$ puts us in precisely the class of functions considered in $\S 6$ of [PU] Some examples are given there of sets $E\left(\frac{1}{2}, \beta\right)$ for which $H D\left(\left(p_{H} \pi\right)_{*} \mu\right)<1$ and $H D\left(E\left(\frac{1}{2}, \beta\right)\right)<D_{B}\left(E\left(\frac{1}{2}, \beta\right)\right)$ They correspond to $\beta$ being the reciprocal of a Pisot number

Finally we shall briefly describe a subclass of self-affine sets (that are graphs of continuous functions) introduced in $[K]$ for which the conjecture stated in the introduction can be proved completely We say that $f I \rightarrow I$ is a self-affine function If there exists $1>H>0$ and an integer $r \geq 4$ such that for every $n \geqq 1,0 \leq \imath<r^{n}$ and $0 \leq h<r^{-n}$ we have

$$
f\left(t r^{-n}+h\right)-f\left(t r^{-n}\right)=T_{n} r^{-n H} f\left(r^{n} h\right)
$$


where $T_{n, 1}$ equals either 1 or -1 It is easy to see that a bounded self-affine function is continuous if and only if the above condition holds for any $0 \leq h \leq r^{-n}$ A useful characterisation of the class $\mathbf{K}$ of self-affine functions for which $f(0)=0, f(1)=1$ can be found in [K] Observe that for $f \in \mathbf{K}$, the graph of $f$ coincides with the self-affine set determined by the contractions

$$
\varphi_{1}(x, y)=\left((l+x) r^{-1}, T_{1, l} r^{-H} y+f\left(r^{-1} l\right)\right), \quad 0 \leq \imath<r
$$

It is easy to check that $D_{B}(\operatorname{graph}(f))=2-H$, and it has been proved in [U] that $H D(\operatorname{graph}(f))=2-H$ if and only if $\left(p_{H} \pi\right)_{*} \mu$ (the probability distribution of $f I \rightarrow \mathbb{R})$ is absolutely contınuous with respect to Lebesgue measure on $[0,1]$

Acknowledgement We would like to thank the referee for pointıng out a mistake in an earlier version of this paper The second author was supported by the A von Humboldt foundation

\section{REFERENCES}

[Be1] T Bedford Hausdorff dimension and box dimension in self-sımılar sets Proc Topology and Measure V, Ernst-Montz-Arndt Universitat Greifswald (1988)

[Be2] T Bedford The box dimension of self-affine graphs and repellers Nonlineanty 2 (1989), 53-71

[BR] T Bohr \& D A Rand The entropy function for characteristic exponents Physica 25D (1986), 387-398

[Bo1] $\mathrm{R}$ Bowen Equilibrium states and the ergodic theory of Anosov diffeomorphisms Lecture Notes in Mathematics 470, Springer (1975)

[Bo2] R Bowen Hausdorff dimension of quas1-circles Publ Math IHES 50 (1979), 11-25

[E] P Erdos On the smoothness properties of a family of Bernoull convolutions Amer $J$ Math 62 (1940), 180-186

[F] K J Falconer The Geometry of Fractal Sets Cambridge University Press, Cambridge, 1985

[H] J E Hutchınson Fractals and self-sımılanty Indiana Univ Math $J$ 30 (1981), 713-747

[K] N Kono On self-affine functions II Japan J Appl Math 5 (1988), 441-454

[MM] A Manning \& H McCluskey Hausdorff dimension for horseshoes Ergod Th \& Dynam Sys 3 (1983), 251-260

[PU] F Przytyckı \& M Urbańskı On the Hausdorff dimension of some fractal sets Studia Math 93 (1989), 155-186

[R] D Ruelle Thermodynamic formalısm the mathematical structures of classical equilibrium statistical mechanics Encyclopedia of Mathematics and its Applications Vol 5, Addison-Wesley (1978)

[U] M Urbańskı The probabılity distribution and Hausdorff dımensıon of self-affine functions Prob Th and Rel Fields 84 (1990), 377-391 